\title{
ldentificação das necessidades do consumidor no processo de desenvolvimento de produtos: uma proposta de inovação ilustrada para o segmento automotivo
}

\author{
Leonardo Ensslin ${ }^{\text {a* }}$, Shirley Queiroz ${ }^{\mathrm{b}}$, Cleci Grzebieluckasc, Sandra Rolim Ensslin ${ }^{\mathrm{d}}$, \\ Elton $\mathrm{Nickel}^{\mathrm{e}}$, Marcos Albuquerque Buson ${ }^{\mathrm{f}}$, Alceu Balbim Junior ${ }^{\mathrm{g}}$ \\ a*leonardoensslin@gmail.com, EPS-UFSC, Brasil \\ bshirleyqueiroz@gmail.com, EPS-UFSC, Brasil \\ cleci@unemat.br, EPS-UFSC, Brasil \\ dsensslin@gmail.com, EPS-UFSC, Brasil \\ eeltonnickel@gmail.com, EPS-UFSC, Brasil \\ fmarcosbuson@gmail.com, EPS-UFSC, Brasil \\ galceubalbim@yahoo.com.br, EPS-UFSC, Brasil
}

\begin{abstract}
Resumo
Dentre as estratégias vencedoras no atual contexto competitivo, emerge a inovação como uma das mais bem sucedidas e, nesta, a capacidade para identificar e mensurar as necessidades dos clientes é possivelmente o maior desafio dos designers. Neste contexto, o presente trabalho sugere uma proposta com o objetivo de ajudar a identificar as necessidades dos clientes e transformá-las em requisitos e características da qualidade, a fim de apoiar o processo decisório na etapa do desenvolvimento do produto. Para testar a proposta, foi criado um cenário e avaliado o grau de atendimento das necessidades de um usuário específico, quando da aquisição de um veículo para uso particular. É apresentado um estudo de caso com fontes de informações primárias não obstrutivas, variáveis quali-quantitativas e a visão de conhecimento construtivista. 0 processo proposto permitiu ao designer identificar e mensurar os requisitos e características da qualidade a partir das necessidades percebidas pelos clientes.
\end{abstract}

Palavras-chave

Design. Requisitos do cliente. MCDA-C. Avaliação de desempenho. Decisão.

\section{Introdução}

A busca por diferenciais competitivos tem privilegiado a função do designer, particularmente quanto à contínua incorporação de inovações nos produtos, serviços e marketing. Inúmeros instrumentos utilizados pelos designers têm sido aperfeiçoados e outros tantos criados, a fim de permitir maior proximidade das necessidades dos usuários. A compreensão de que não basta inovar na funcionalidade do produto, se esta não for percebida pelo usuário como agregadora de valor, tem incentivado as instituições científicas a aperfeiçoarem seus instrumentos de identificação, organização e mensuração das necessidades dos usuários. Um desses instrumentos da Área científica é conhecido como Avaliação de Desempenho, que tem por objetivo promover um melhor entendimento das necessidades dos usuários, sem descuidar das características de qualidade do produto para atender a suas funções técnicas.

Isto significa que para desenvolver e lançar um novo produto no mercado é de fundamental importância conhecer os desejos, expectativas e as necessidades do segmento alvo para o qual o produto será desenvolvido. 
Para conhecer as expectativas do segmento-alvo é necessário responder a questões do tipo: (i) como identificar as necessidades do consumidor?; (ii) Como organizar as necessidades em classes equivalentes?; Como mensurá-las?; Como integrá-las?; e Como utilizar todo este conhecimento para aperfeiçoar o produto?

0 objetivo do presente trabalho é: propor um processo que permita construir um modelo para avaliar o grau de atendimento das necessidades de três usuários, quando da aquisição de um veículo para uso particular. Sua expansão para captar as necessidades de uma população poderá ser realizada por meio de procedimentos como proposto por Ensslin, Montibeller e Noronha (2001) para decisões em grupo.

A necessidade de modelos científicos que explicitem os critérios que representam as percepções de valor e preferência dos usuários, com base em processos sistêmicos de avaliação de desempenho, adicionado das incertezas e imprecisões quanto a seus valores e preferências e sua singularidade, orienta o processo para a definição do instrumento a ser utilizado como construtivista (ENSSLIN et al., 2010).

A metodologia MCDA-C, por sua capacidade de ajudar o decisor a expandir seu conhecimento do problema em termos da identificação, organização, mensuração local, explicitação dos níveis de desempenho de referência, integração e aperfeiçoamento da performance do produto, é o instrumento recomendado para estas situações. Pelas razões expostas, a metodologia MCDA-C será o instrumento de pesquisa a ser utilizado, neste trabalho.

0 presente artigo descreve um estudo de caso no qual foi desenvolvido um modelo personalizado para um cenário constituído por uma amostra de três usuários de automóveis, com particularidades específicas. Sua generalidade requer a realização de trabalhos similares com outras amostras e suportados por testes estatísticos que atestem representatividade. 0 propósito desta pesquisa é evidenciar como fazer e quais as potencialidades do uso do processo proposto.

A fonte de coleta dos dados é de natureza primária, pelo fato de os dados terem sido coletados diretamente junto aos usuários da amostra, via entrevistas semiestruturadas. A abordagem metodológica utilizada pode ser classificada como quali-quantitativa. Qualitativa na estruturação quando identifica os critérios, construção das escalas ordinais e quando das Recomendações. Quantitativa quando transforma as escalas ordinais em cardinais, permitindo a mensuração cardinal da performance local, e sua integração, permitindo a mensuração cardinal da performance global. A lógica da pesquisa é mista, indutiva na etapa da Estruturação e dedutiva na Avaliação.

A relevância da pesquisa pode ser argumentada em termos das contribuições (i) teóricas; e (ii) gerenciais que ela pretende oferecer. Com relação a (i), a proposta teórico-metodológica, ao oferecer um processo estruturado para identificar oportunidades para o desenvolvimento de um produto segundo a percepção de seus usuários em uma forma participativa; e (ii), ao oferecer um instrumento que permite ao decisor desenvolver seu entendimento do contexto de forma a permitir-lhe explicitar seus valores (critérios) e preferências (funções de valor e taxas de compensação) e as consequências de suas decisões.

0 presente trabalho, além desta introdução, contém um referencial teórico, Construção do modelo MCDA - C para avaliar as características de um automóvel, Considerações finais e referências bibliográficas.

\section{Referencial teórico}

Nesta seção, encontra-se o referencial teórico abordando o Processo de Desenvolvimento de Produtos e a Metodologia Multicritério.

\subsection{PDP e necessidades dos clientes}

0 processo de desenvolvimento de produtos (PDP) envolve uma série de fases, tarefas e atividades que se complementam, variam entre simultâneas e sequenciais e apresentam suas especificidades de acordo com o produto a ser desenvolvido. A partir dessa definição, observa-se que desenvolver produtos é uma atividade complexa e singular, com objetivos conflitantes e não bem entendidos pelos atores, que necessita ser monitorada e gerenciada para que um novo produto seja bem sucedido no mercado competitivo.

Essa complexidade faz com que a busca para aprimorar e tornar o processo de desenvolvimento de produtos mais eficiente e eficaz seja constante e proporcione uma gama de metodologias que ofereçam suporte teórico, recomendem procedimentos e forneçam técnicas e ferramentas úteis nas diversas fases do projeto.

0 ciclo de vida do produto é dividido em etapas de pré-desenvolvimento, desenvolvimento e pós-desenvolvimento. A Figura 1 apresenta as etapas do PDP divididas em oito fases que 
Ciclo de desenvolvimento do produto

Pré

Figura 1. Ciclo de vida do produto. Fonte: Adaptado de Larson e Gobeli (1988); Rozenfeld et al. (2006).

representam a evolução, a complexidade e alocação de recursos no processo de desenvolvimento de um produto em que, na fase de Lançamento do Produto é praticamente proibitiva uma mudança de escopo do produto ou projeto (LARSON; GOBELI, 1988; ROZENFELD et al., 2006).

As diversas propostas metodológicas têm como objetivo principal apoiar a realização das atividades necessárias ao desenvolvimento de produtos que proporcionem retorno máximo tanto para seus usuários, quanto para seus stakeholders. Contudo, seja qual for o método adotado, elas são utilizadas como apoio ao processo projetual, auxiliando, por meio de técnicas e ferramentas, o planejamento, coleta, análise e a execução das etapas ao longo do processo. Deste modo, o sucesso na aplicação desses métodos irá sempre depender da capacidade técnica e criativa de quem os utiliza (BOMFIM, 1995).

Apesar de apresentarem suas especificidades, os modelos disponíveis para o desenvolvimento de novos produtos compartilham os mesmos interesses (objetivos) e apresentam recomendações comuns, com conceitos e objetivos similares, os quais são identificados, direcionados e valorados pelo consumidor. Fatores que irão garantir a sobrevivência do produto em um mercado cada vez mais competitivo.

Considerando que todo produto é projetado para ser utilizado por ou para alguém, as propostas metodológicas recomendam que sejam tomados cuidados especiais em termos de dados e que estes estejam alinhados com as necessidades do segmento-alvo deste produto. Estabelecido o público alvo, o passo crítico para o sucesso do produto é a identificação de suas necessidades. Estas são transformadas em requisitos que serão utilizados para orientar o desenvolvimento do produto. Desta forma, a adequação do produto será uma função de quão bem as necessidades foram identificadas e representadas por seus requisitos. 0 presente trabalho propõe contribuir nesta área, isto é, apresentar um processo para auxiliar na identificação e representação na forma de escalas os requisitos para desenvolver e/ou gerenciar a performance de um produto.

De acordo com Kindlein Junior (2002, p. 01)

[...] a pesquisa e a metodologia necessárias para conhecer o desejo do cliente e do empresariado ainda é compilada, na sua grande parte, pela intuição do designer que usa dados levantados, tendências e procedimentos técnicos assegurando assim o caminho escolhido para o projeto $\mathrm{e}$ minimizando riscos dos investimentos aplicados.

Portanto, esta fase denominada Projeto Informacional transforma os dados das necessidades dos clientes em um conjunto de informações que gera uma lista de especificações na forma de requisitos que serão utilizados na fase de Projeto Conceitual.

Atualmente o levantamento de informações sobre o segmento-alvo é feito principalmente por meio da aplicação de questionários, entrevistas e focus group, que são aplicados em pessoas previamente selecionadas de acordo com o perfil do público-alvo. Após a coleta de dados obtidos nessa fase, pode-se criar um personagem símbolo, que represente o perfil do usuário do produto a ser desenvolvido, creating a persona (BURDEK, 2006). A criação desse personagem símbolo é feita para que a equipe de desenvolvimento tenha uma imagem mais concreta e humanizada do perfil do consumidor do novo produto.

0 foco desse trabalho reside no levantamento dos desejos e necessidades do público-alvo de um produto a ser desenvolvido, os quais foram obtidos por meio da participação de um decisor, selecionado a partir da criação de um personagem símbolo. Para identificar as necessidades desse personagem, utilizou-se um modelo multicritério como instrumento para avaliar de modo mais preciso suas necessidades em relação a um automóvel e convertê-las em requisitos mensuráveis para o design desse automóvel.

\subsection{A abordagem multicritério}

0 instrumento de intervenção adotado foi a Metodologia Multicritério de Apoio à Decisão 
- Construtivista (MCDA-C) que tem como principal propósito expandir o conhecimento no decisor sobre contextos complexos, conflituosos e incertos. A MCDA-C utiliza instrumentos de estruturação de informações capazes de permitir explicitar a compreensão das consequências de decisões nos aspectos que o decisor julga relevantes. Dessa forma, a metodologia apresentada é um instrumento recomendado em casos em que o decisor precisa atuar com consistência, fundamentação e transparência para negociar com todos os atores envolvidos sobre suas decisões (ENSSLIN; DUTRA; ENSSLIN, 2000).

A MCDA-C encontra suas mais remotas origens há mais de dois séculos. Contudo, sua consolidação como instrumento científico de gestão ocorre somente a partir da década de 1980 com os trabalhos de Roy (2006) e Landry (1995) ao definir os limites da objetividade para os processos de apoio à decisão, de Skinner (1986) e Keeney (1992) ao reconhecer que os atributos (objetivos, critérios) são específicos ao decisor em cada contexto, de Bana e Costa (1999) ao explicitar as convicções da MCDA, dentre outros.

A MCDA-C surge como uma ramificação da MCDA tradicional para apoiar os decisores em contextos complexos, conflituosos e incertos. Complexos por envolverem múltiplas variáveis qualitativas e quantitativas, parcialmente ou não explicitadas. Conflituosos por envolverem múltiplos atores com interesses não necessariamente alinhados e/ou com preocupações distintas do decisor que não tem interesse de confrontá-los, mesmo reconhecendo que estes estarão disputando os escassos recursos. Incertos por requererem o conhecimento de informações qualitativas e quantitativas que os decisores reconhecem não saber quais são e/ou a forma de mensurá-los, mas que desejam desenvolver este conhecimento para poder tomar decisões conscientes, fundamentadas e segundo seus valores e preferências (ZIMMERMANN, 2000).

Existem diferenças significativas entre as duas abordagens. Enquanto a MCDA procura encontrar a solução ótima de um modelo cujos objetivos podem ser obtidos externos ao contexto e/ou solicitando ao decisor que os explicite e, portanto, sem um processo formal de estruturação, a MCDA-C centra seus esforços no processo de estruturação visando a geração de conhecimento aos decisores (ENSSLIN; MONTIBELLER; NORONHA, 2001).

Tais características são evidenciadas por Ensslin, Dutra e Ensslin (2000), os quais acreditam que a força da MCDA-C torna-se evidente em situações complexas, nas quais existe uma combinação de diferentes atores, cada um com seus próprios valores, percepções, objetivos, interagindo em relações assimétricas de poder.

\subsubsection{Metodologia Multicritério de Apoio à Decisão Construtivista (MCDA-C)}

A metodologia Multicritério de Apoio à Decisão Construtivista (MCDA-C) também denominada de abordagem Soft PO (ENSSLIN; ENSSLIN; PETRI, 2007; GOMES; GOMES, 2007) deve ser entendida como uma ciência que busca desenvolver uma rede de conceitos, modelos, procedimentos e resultados capazes de formar estruturas que possam atuar como um conjunto de hipóteses a fim de guiar os decisores, mantendo a coerência de acordo com seus objetivos e valores (ENSSLIN; ENSSLIN; PETRI, 2007). Gomes e Gomes (2007) apontam que a abordagem Soft P0 tem como principal função estruturar o problema antes de tentar resolvê-lo.

A metodologia MCDA-C consiste em três fases diferentes, porém, correlacionadas às quais podem ser mais bem visualizadas na Figura 2 .

A fase de estruturação objetiva estabelecer um mecanismo de comunicação entre os vários atores envolvidos que irão promover um entendimento e

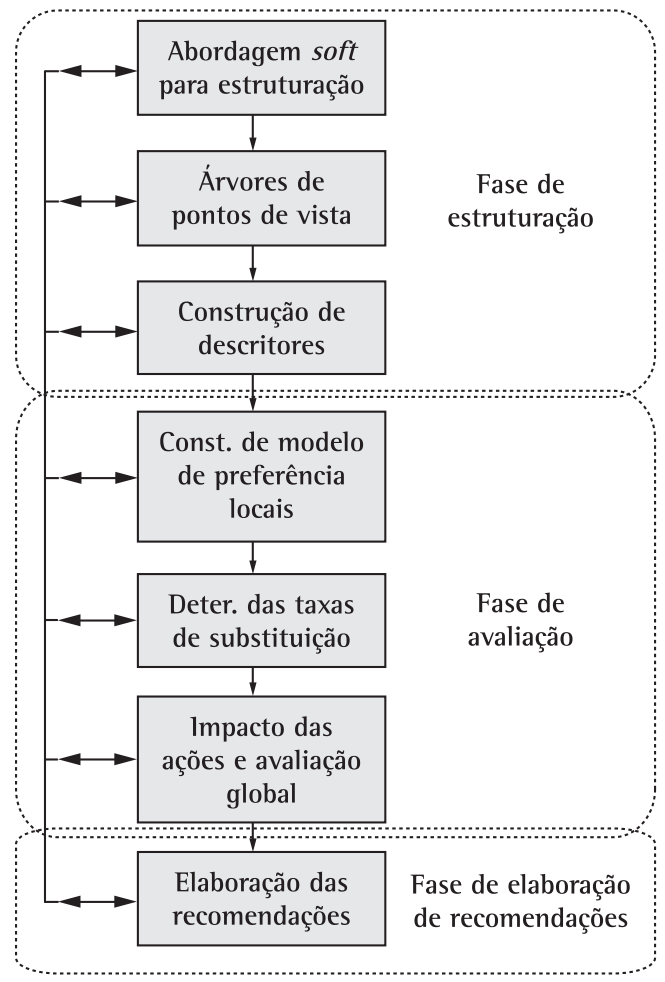

Figura 2. Fases do aprendizado gerado pela metodologia MCDA-C. Fonte: Ensslin (2002, p.156) adaptado de Ensslin et al. (2000). 
aprendizado comum entre eles (ENSSLIN; DUTRA; ENSSLIN, 2000). Bana e Costa et al. (1999) acreditam que a fase da estruturação representa aproximadamente $80 \%$ do esforço requerido para desenvolver o conhecimento que permita chegar à solução. Esta fase busca identificar os objetivos, caracterizar os fatos considerados relevantes no processo de apoio, avaliar e identificar as alternativas viáveis, entre outras etapas que dependem de cada problema específico. Nesta fase, são realizadas basicamente as seguintes etapas: a) identificação do problema; b) árvore de pontos de vista; c) construção dos descritores para a medição do desempenho das alternativas potenciais em cada ponto de vista fundamental (PVF). Para identificar o problema Ensslin; Ensslin e Petri (2007) destacam que é necessário ter claro, quem é o decisor, qual a insatisfação, a relevância do problema e a possibilidade de resolução (factibilidade).

Neste contexto, entende-se por pontos de vista fundamentais (PVF) as dimensões consideradas pelo decisor como necessárias e suficientes para avaliar as ações potenciais. A Árvore dos pontos de vista fundamentais - consiste na organização dos PVFs em uma estrutura arborescente. Os PVFs são dimensões preferencialmente independentes com desmembramentos em PVEs que são mensurados via descritores (escalas ordinais).

Descritor - é um conjunto de níveis de impacto que serve como base para medir o desempenho das ações potenciais de cada Ponto de Vista. Os descritores são escalas ordinais e podem ser quantitativos ou qualitativos (ENSSLIN; MONTIBELLER; NORONHA, 2001; PARATH et al., 2005).

As etapas da fase de avaliação consistem na construção de modelos que expressem as preferências e os juízos de valor do decisor, a identificação dos perfis de impacto das ações alternativas, geração de eventuais ações e a definição do modelo de avaliação global.

A etapa das recomendações procura fornecer subsídios aos decisores por meio de ferramentas (conceitos, modelos e procedimentos) para que esses tenham condições de analisar e escolher qual a estratégia mais adequada a ser adotada (LIMA, 2003) e promover um debate a respeito das oportunidades de aperfeiçoamento que o modelo gerou (ENSSLIN; ENSSLIN; PETRI, 2007).

\section{Construção do modelo para avaliação do desempenho}

Em situações complexas, conflituosas e incertas como a do presente estudo, que envolvem múltiplos e conflitantes critérios parcialmente conhecidos, metodologias do tipo multicritério, que reconhecem os limites da objetividade, emergem como o instrumento de intervenção recomendado (ROY, 1993, 2006; ROY; VANDERPOOTEN, 1996).

0 modelo MCDA-C proposto tem como objetivo servir de ferramenta para o desenvolvimento do entendimento do decisor sobre as consequências que as propriedades das possiveis alternativas exercem em seu sistema de valor. Este conhecimento permitirá que os responsáveis pelo design destes produtos possam conhecer as demandas dos usuários e em que intensidade estão ou não sendo atendidas.

\subsection{Estruturação do modelo}

Esta fase tem por objetivo explicar o contexto e construir um grau de entendimento do ambiente que permita delimitar o que pertence ao problema e o que está fora. Inicia-se pela identificação do público alvo e, dentre estes, qual a amostra de atores que o representará. Seguem-se entrevistas realizadas com instrumentos como: brainstormig (ROY, 2006), mapas cognitivos (EDEN, 1988), cluster (KEENEY, 1992), e Teoria da Mensuração (ROBERTS, 1979; BARZILAl, 2001; AZEVEDO, 2001). Ao final desta etapa, tem-se identificados, organizados e mensurados os requisitos percebidos como necessários e suficientes para avaliar o produto, segundo a visão dos clientes da amostra (ENSSLIN; MONTIBELLER, NORONHA, 2001).

\subsubsection{Contextualização, subsistema de atores e rótulo}

0 Estudo de Caso foi desenvolvido em Florianópolis, SC, tendo como público-alvo mulheres independentes, com filhos pequenos e vida pessoal e profissional ativa.

Com base nesse perfil, foi selecionado como decisor (a) um conjunto de três mulheres com estas características a fim de que identificassem quais as características julgadas por elas como importantes no momento da aquisição de um automóvel.

A estruturação do problema foi realizada por meio de um brainstorming. Os facilitadores (equipe de projeto) se preocuparam em criar condições para que as decisoras pudessem falar o mais abertamente possível e com o mínimo de interferência sobre tudo que valora e as preocupa no contexto.

A análise concentrou-se nas características de design de um automóvel e no papel delas, no intuito de suprir as necessidades dos decisores, inserindo os requisitos por elas tidos como importantes, no 
desenvolvimento do novo veículo ou em veículos já disponíveis.

Como o conhecimento do decisor é personalizado, é relevante iniciar o processo pela identificação dos atores. Neste caso os atores foram:

Decisores:

- Grzebieluckas, Cleci;

- Ensslin, Sandra;

- Queiroz, Shirley Gomes.

Profissionais liberais com intensa vida profissional.

Facilitadores:

- Balbim, Alceu Junior;

- Buson, Marcos Albuquerque;

- Ensslin, Leonardo;

- Nickel, Elton.

Engenheiros especialistas em estruturação e/ou design de produtos.

Intervenientes:

- Parentes.

Agidos:

- Amigos.

Como rótulo os decisores acordaram:

- Avaliação da adequacidade de automóveis para atender às necessidades de mulheres independentes e com filhos.

Ao trabalhar em contato direto com o cliente (decisor), procura-se incentivá-lo a explicitar suas necessidades, restrições, valores e preferências. A metodologia MCDA-C possui um processo estruturado que lhe permite expandir e organizar o entendimento do decisor para que este consiga ter a compreensão de quais as consequências que ele considera relevante neste problema específico. Esgotadas as oportunidades ordinais de aperfeiçoamento do conhecimento do decisor, a MCDA-C transforma as informações ordinais em cardinais, solicitando, ao decisor, as informações sobre as diferenças de atratividade. Esta etapa é denominada Avaliação e permite desenvolver uma representação matemática que expressa numericamente o quanto uma dada alternativa atende a cada uma de suas necessidades individuais e globais no contexto sendo estudado. A partir deste momento o processo para gerar alternativas de solução que atendam efetivamente as suas expectativas e necessidades referentes a um automóvel é imediato.

\subsubsection{Elementos primários de avaliação, conceitos e áreas de preocupação}

Para o levantamento dos dados relativos ao sistema de valores, foram gravadas entrevistas abertas com os decisores. Nas entrevistas, foi solicitado que os decisores discorressem a respeito do problema. Suas declarações foram analisadas para identificar os Elementos Primários de Avaliação (EPAs), que são as características ou propriedades do contexto que o decisor julga que impactam seus valores. 0 maior número possível de EPAs deve ser identificado e novos podem surgir com a combinação dos pré-existentes. A análise das entrevistas permitiu a identificação inicial de 60 EPAs.

A partir de cada EPA seu entendimento foi expandido transformando-o em conceito orientado para a ação. Um conceito expressa a direção de preferência associada ao EPA juntamente com seu polo psicológico oposto. Sua construção é realizada estabelecendo-se com o decisor o objetivo subjacente ao EPA e seu oposto psicológico. Por exemplo, o conceito relacionado ao EPA Ajuste do Retrovisor. Permitir que o ajuste do retrovisor seja feito por meio de controle remoto... ajuste manual representado na Figura 3 ( [...] leia-se "ao invés de").

Tendo em vista que todos os EPAs e respectivos conceitos orientados para ação foram gerados a partir de um processo de brainstorming, encontram-se dispostos de forma aleatória e, aparentemente, não apresentam ligações diretas. 0 passo seguinte consiste em organizar os conceitos em áreas de preocupação. Foram identificadas duas grandes áreas de preocupação. Uma relacionada a fatores práticos e outra a fatores estéticos e simbólicos. 0 primeiro foi subdividido em segurança e conforto e o segundo foi subdividido em status, estética e estilo. Por fim, a subárea segurança foi dividida em estabilidade e proteção e a subárea conforto foi dividida em acionamentos, espaço e opcionais. Esses agrupamentos geraram oito Pontos de Vista Fundamentais - PVFs: estabilidade, proteção, acionamentos, espaço, opcionais, status, estética e estilo conforme apresentado na Figura 4.

Esta atividade esgotou o potencial de construção de conhecimento do brainstorming. Para continuar o processo de expansão do entendimento do contexto, a MCDA-C vale-se agora dos mapas cognitivos. Para cada PVF, foi desenvolvido um estrutura de mapa cognitivo. A Figura 5 demonstra a construção do mapa cognitivo acionamentos e a exploração dos conceitos na busca dos valores e dos meios para alcançar os objetivos dos decisores. Com o mapa pronto, foram definidos os ramos, realizadas as análises de redundâncias e os Pontos de Vista elementares. Após a construção desse mapa, foram identificados os seguintes PVEs: ajuste do retrovisor, controle para portas (dividido em dois sub PVEs: porta-malas e portas dianteiras), conforto térmico, consumo e limpador de para-brisas. 
0 mesmo procedimento foi adotado para a construção das Árvores de Valor em todos os demais PVFs.

Para cada PVE da estrutura arborescente são então construídas as escalas ordinais denominadas Descritores que permitem mensurar ordinalmente o alcance do respectivo Ponto de Vista. Os Descritores servem para medir os possíveis níveis de impacto de uma determinada ação. Para cada descritor, foi solicitado aos decisores que identificassem as fronteiras entre os níveis julgados como em nível de excelência, nível de mercado, e nível comprometedor. 0 nível na fronteira superior é denominado Bom e o inferior Neutro (ENSSLIN; DUTRA; ENSSLIN, 2000). Estes são os níveis de referência do descritor.

A Figura 6 a seguir apresenta o conjunto de descritores relacionados ao PFV acionamentos e a situação atual (de acordo com os decisores).
Ao concluir a construção da Estrutura Hierárquica de Valor com os Descritores, desenvolveu-se todo o entendimento do contexto permitido por uma estrutura não numérica (escalas nominais e ordinais). Para continuar o processo de desenvolvimento do entendimento, devem ser incorporadas informações que possibilitem transformar as escalas ordinais em cardinais, que é o propósito da etapa seguinte da metodologia MCDA-C.

\subsection{Avaliação}

Ao concluir a etapa de estruturação, a metodologia MCDA-C terá construído um modelo contendo os aspectos julgados pelo(s) decisor(es) como necessários e suficientes para avaliar o contexto. As escalas neste modelo são ordinais e denominadas Descritores, conforme Figura 6. As referidas escalas, muitas vezes, se valem de símbolos numéricos para sua representação, estes, no entanto,

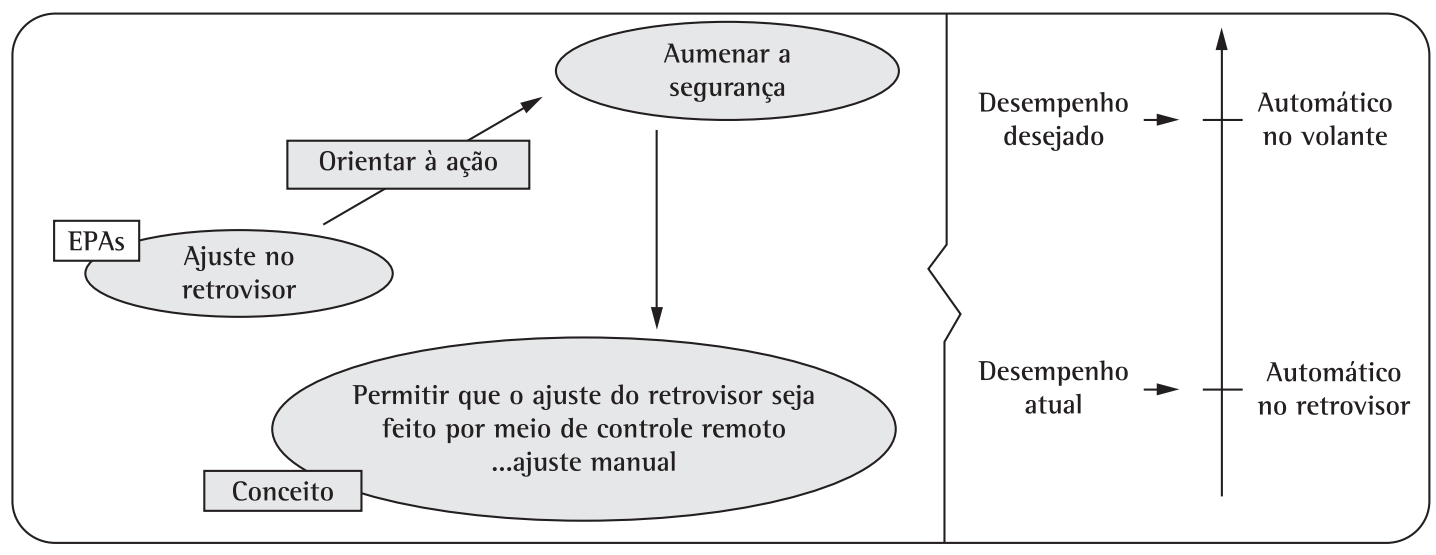

Figura 3. Construção dos conceitos. Fonte: Adaptado de Ensslin, Montibeller e Noronha (2001).

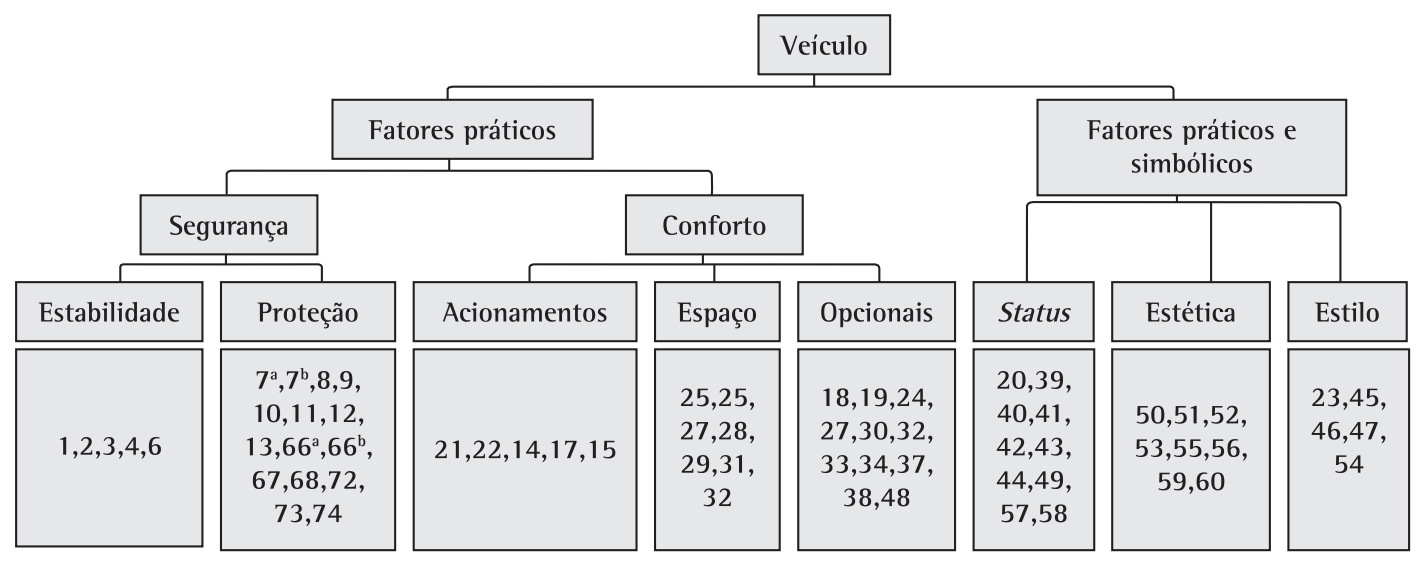

Figura 4. Estrutura arborescente do modelo com PVFs. 


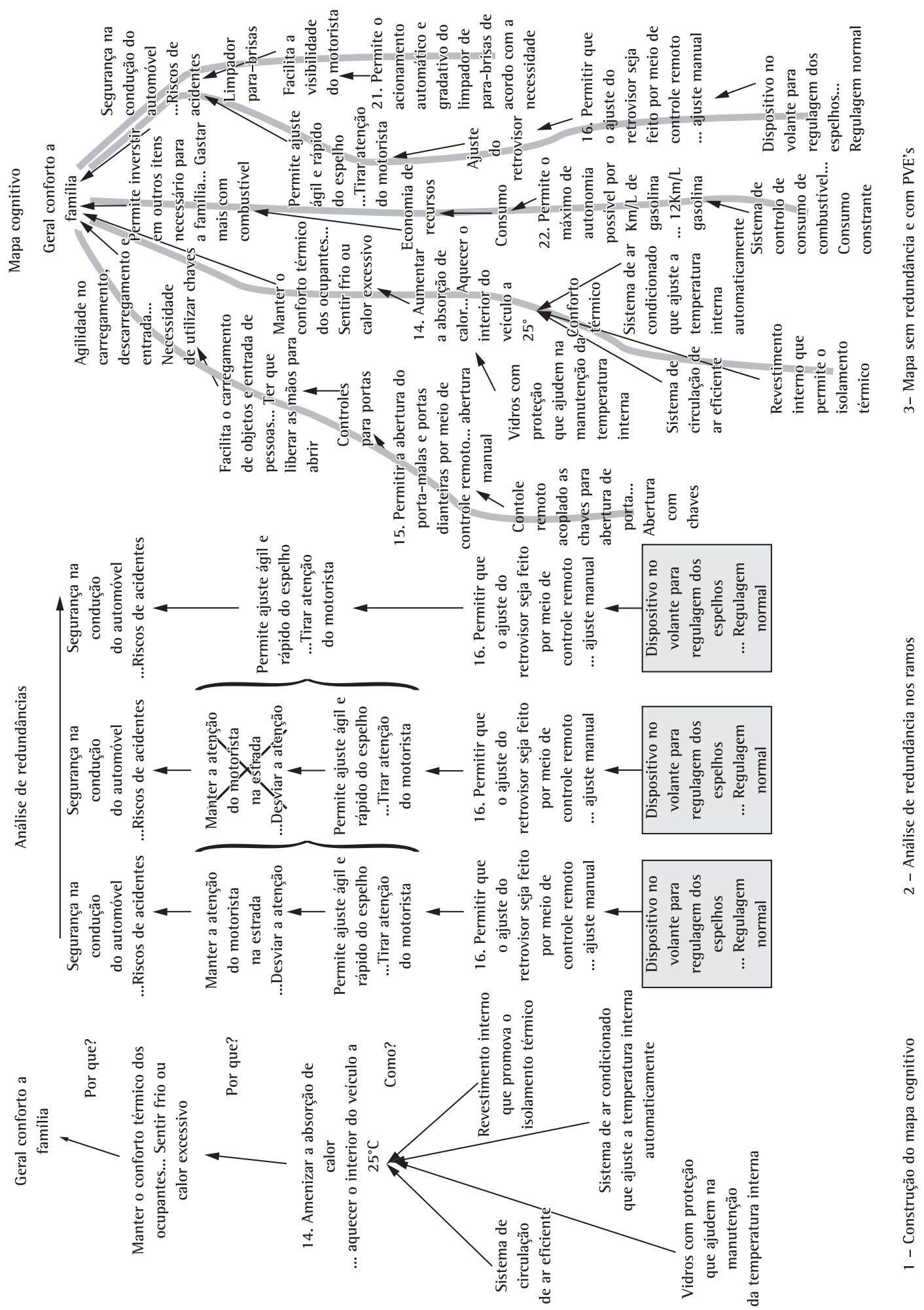

Figura 5. Mapa de relações meio-fim e transição para árvore de valores. 


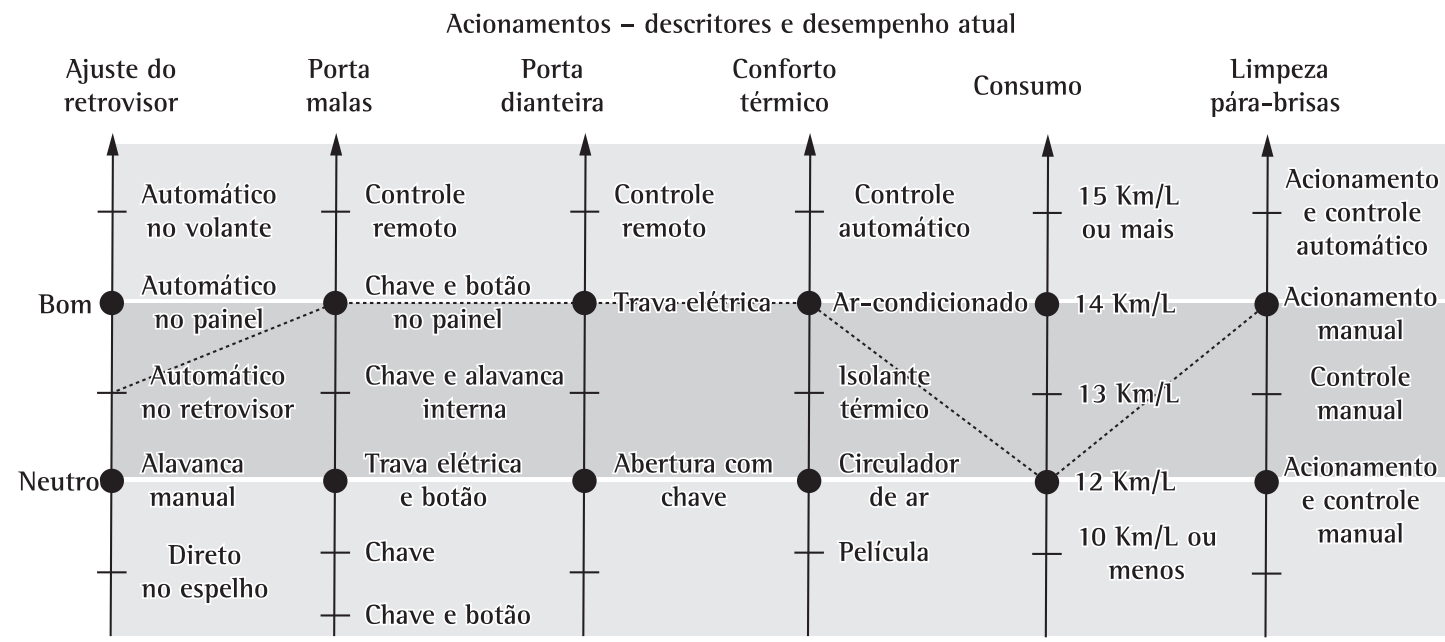

Figura 6. Descritores dos PVEs de acionamentos e representação do Status Quo do decisor.

são simplesmente símbolos alfanuméricos, e não números do conjunto $\Re^{n}$ (conjunto dos números reais) - para mais detalhes, ver Ensslin, Montibeller e Noronha (2001), Barzilai (2001) e Azevedo (2001).

A MCDA-C reconhece as diferenças entre as escalas ordinais e cardinais e, para realizar a transformação, necessita mais uma vez a participação do decisor para fornecer informações que permitam conhecer a diferença de atratividade entre os níveis de cada escala. Esta atividade pode ser realizada por variados métodos, tais como: Pontuação Direta, Bissecção, MACBETH, dentre outras (ENSSLIN; MONTIBELLER; NORONHA, 2001). A MCDA-C vale-se de todos estes métodos para transformar as escalas ordinais em cardinais. 0 método MACBETH, foi utilizado neste trabalho por sua fundamentação teórica, representatividade e reconhecimento prático, e por ser o mais empregado.

Destaque-se que o MACBETH - Measuring Attractiveness by a Cathegorical Based Evaluation Technique é unicamente um método para transformar escalas ordinais em cardinais a partir de juízos absolutos sobre a diferença de atratividade entre duas alternativas. Na metodologia de apoio à decisão, como pode ser evidenciado em Bana e Costa, De Corte e Vansnick, (2005, p. 437):

\section{[...] The MACBETH approach and the M-MACBETH software have been used to derive preference scales or value functions and scaling constants in many public and private applications of multicriteria additive value analysis, some of them reported in the literature.}

\subsubsection{Funções de valor}

Obtidos os Descritores de todos os pontos de vista, a metodologia MCDA-C, seguindo seu propósito de construir o entendimento do(s) decisor(es), solicita que informe $(\mathrm{m})$ a diferença de atratividade entre os níveis dos descritores (escalas ordinais). A partir destas informações, com a ajuda do software M-Macbeth, constroem-se escalas cardinais que atendam os juízos de preferências do decisor. Estas escalas denominam-se Funções de Valor.

0 procedimento de uso do método MACBETH consiste em solicitar ao decisor que expresse a diferença de atratividade entre duas alternativas potenciais a e b (a mais atrativa que b) com base em uma escala ordinal de sete categorias semânticas propostas a priori ao decisor para cada intervalo do descritor (BANA E COSTA et al., 1999). Os níveis de atratividade da escala semântica são: nula, muito fraca, fraca, moderada, forte, muito forte e extrema. Em seguida, são estabelecidos os Níveis de ancoragem Bom (100) e Neutro (0), transformando a escala em uma Escala de Intervalos Ancorada. Deste modo, os Níveis Âncora Bom e Neutro terão igual grau de atratividade e igual pontuação numérica para todas as funções de valor.

Com base nas respostas do decisor, constrói-se a Matriz de Julgamentos, cujos valores servem de entrada para o software determinar a função de valor.

A Figura 7 a seguir apresenta os julgamentos segundo a percepção dos decisores. Inicialmente é construída uma Função de Valor que depois é transformada em outra equivalente com a pontuação 100 (cem) no Nível Bom e 0 (zero) no Nível Neutro. Esta transformação de escalas promovida pela incorporação dos Níveis Bom e Neutro é fundamental para: (i) testar a independência 


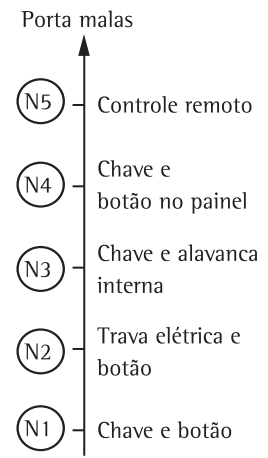

\begin{tabular}{|c|c|c|c|c|c|c|c|}
\hline \multicolumn{8}{|c|}{ Matriz de diferenças de atratividade para o PV - porta malas } \\
\hline & & & N5 & N4 & N3 & N2 & $\mathrm{N} 1$ \\
\hline & N5 & $\begin{array}{l}\text { Controle } \\
\text { remoto }\end{array}$ & & Moderada & Forte & $\begin{array}{l}\text { Muito } \\
\text { forte }\end{array}$ & Extrema \\
\hline \multirow[t]{2}{*}{ Bom } & N4 & $\begin{array}{c}\text { Chave } \\
\text { botão no painel }\end{array}$ & & & $\begin{array}{l}\text { Muito } \\
\text { fraca }\end{array}$ & Moderada & $\begin{array}{l}\text { Muito } \\
\text { forte }\end{array}$ \\
\hline & N3 & $\begin{array}{c}\text { Chave e alavanca } \\
\text { interna }\end{array}$ & & & & Fraca & Forte \\
\hline \multirow[t]{2}{*}{ Neutro } & N2 & $\begin{array}{c}\text { Trava elétrica e } \\
\text { botão }\end{array}$ & & & & & Forte \\
\hline & N1 & Chave e botão & & & & & \\
\hline
\end{tabular}

Escala cardinal ou

função de valor

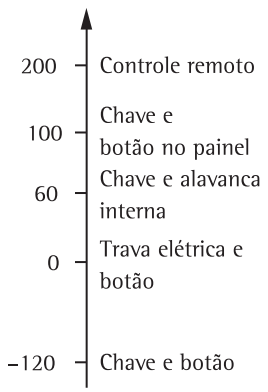

Figura 7. Transformação da escala ordinal do descritor na escala cardinal do sub PVE - porta-malas.

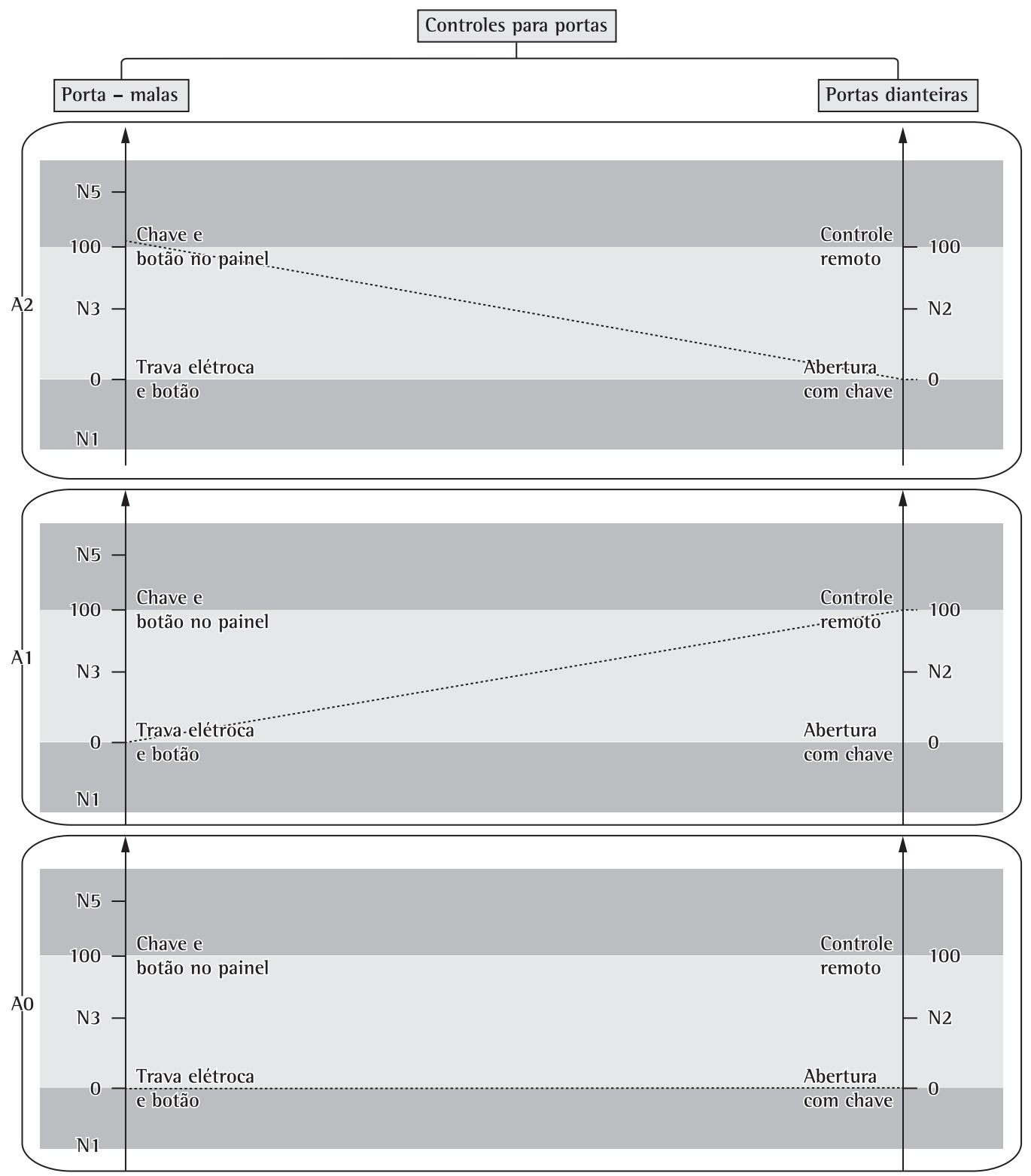

Figura 8. Alternativas fictícias para a determinação das taxas de compensação para o PVE - Controle Para Portas. 
preferencial dos Pontos de Vista; (ii) permitir a determinação das taxas de compensação para a construção do Modelo Global de Avaliação.

Uma vez construídas as Funções de Valor para todos os descritores do modelo, é necessário integrar os pontos de vista, a fim de poder visualizar o desempenho global. Esta atividade é realizada por meio da determinação das taxas de compensação. 0 procedimento utilizado para esse fim é propor alternativas fictícias ao decisor com base no julgamento feito em relação à diferença de atratividade global entre as alternativas.

\begin{tabular}{|c|c|c|c|c|c|}
\hline & A1 & A2 & A0 & Soma & Ordem \\
\hline $\mathrm{A} 1$ & $\times$ & 1 & 1 & 2 & $1^{\circ}$ \\
\hline $\mathrm{A} 2$ & 0 & $\times$ & 1 & 1 & $2^{\circ}$ \\
\hline $\mathrm{A} 3$ & 0 & 0 & $\times$ & 0 & $3^{\circ}$ \\
\hline
\end{tabular}

Figura 9. Matriz de Roberts para hierarquizar as alternativas.
Por exemplo, o PVE controle para portas possui dois Sub PVEs, porta-malas e portas dianteiras, que necessitam ter suas taxas determinadas a fim de se identificar a contribuição que cada uma delas exerce em seu PVE superior. Inicialmente, evidenciam-se as alternativas fictícias apresentadas na Figura 8.

A seguir, ordenam-se as alternativas, o que pode ser realizado utilizando-se a Matriz de Roberts, conforme apresentado na Figura 9. Para isso, o decisor deverá fornecer as informações sobre as alternativas potenciais construídas segundo seu juízo preferencial. Sempre que preferir a alternativa da linha, marca-se na coluna com a qual se está comparando o valor 1 , em caso contrário, zero. Ao final, somam-se os valores das linhas e se obtém o grau de preferência conforme o valor da soma. Gera-se, desta forma, a hierarquização das alternativas, cuja ordem reflete a preferência do decisor para passar do nível Neutro para o nível Bom em cada sub PVE.

Uma vez hierarquizadas as alternativas o decisor fornece a intensidade preferencial das diferenças de atratividade entre elas e por meio do software

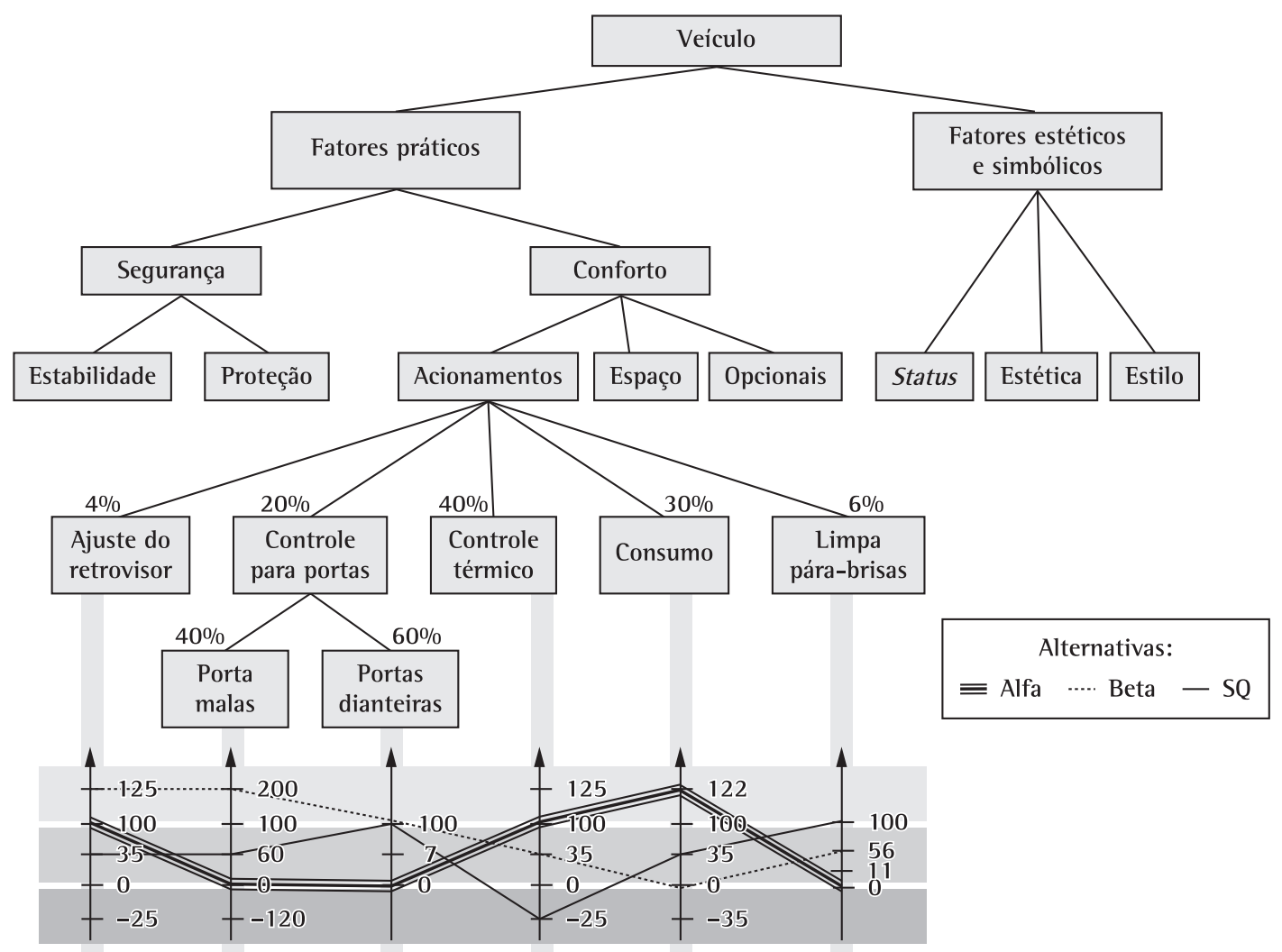

Figura 10. Estrutura arborescente do PVF acionamentos com seus respectivos PVEs, suas taxas de substituição, e perfil das alternativas alfa, beta e SQ - Status Quo. 
MACBETH (BANA E COSTA; DE CORTE; VANSNICK; 2005) determinam-se suas taxas. Assim, a taxa pertencente ao ponto de vista portas dianteiras foi de $60 \%$, enquanto que a taxa pertencente ao ponto de vista porta-mala foi de 40\%. 0 mesmo procedimento foi efetuado para todo o modelo, pontualmente para cada conjunto de pontos de vista. Como exemplo, a Figura 10, apresenta as taxas obtidas para o PVF acionamentos. Como pode ser observado, para o processo utilizado, a taxa corresponde à contribuição do ponto de vista quando a performance passa do nível Neutro para o nível Bom.

Tendo definido as taxas equivalentes a cada ponto de vista, foi possível elaborar a equação global do modelo a fim de que os decisores, além de avaliar localmente o desempenho das ações potenciais, pudessem melhor comparar as alternativas existentes assim como as criadas por eles com base em suas performances globais. $\mathrm{Na}$ Figura 11, um recorte da equação que abrange o ponto de vista acionamentos está em destaque.

$$
\mathrm{V}_{\text {Acion }}(\mathrm{a})=0,04\left[\begin{array}{c}
165 \\
100 \\
40 \\
0 \\
-30
\end{array}\right]+0,2\left\{0,4\left[\begin{array}{c}
200 \\
100 \\
60 \\
0 \\
-120
\end{array}\right]+0,6\left[\begin{array}{c}
100 \\
75 \\
0
\end{array}\right]\right\}+0,4\left[\begin{array}{c}
125 \\
100 \\
35 \\
0 \\
-25
\end{array}\right]+0,3\left[\begin{array}{c}
122 \\
100 \\
55 \\
0 \\
-35
\end{array}\right]+0,06\left[\begin{array}{c}
100 \\
56 \\
11 \\
0
\end{array}\right]
$$

Figura 11. Representação algébrica do PVF - Acionamentos.

$$
\begin{gathered}
\mathrm{V}_{\text {Acion }} \text { (Alfa) }=0,04\left[\begin{array}{c}
165 \\
100 \\
40 \\
0 \\
-30
\end{array}\right]+0,2\left\{0,4\left[\begin{array}{c}
200 \\
100 \\
60 \\
0 \\
-120
\end{array}\right]+0,6\left[\begin{array}{c}
100 \\
75 \\
0
\end{array}\right]\right\}+0,4\left[\begin{array}{c}
125 \\
100 \\
35 \\
0 \\
-25
\end{array}\right]+0,3\left[\begin{array}{c}
122 \\
100 \\
55 \\
0 \\
-35
\end{array}\right]+0,06\left[\begin{array}{c}
100 \\
56 \\
11 \\
0
\end{array}\right] \\
\mathrm{V}_{\text {Acion }}(\text { Alfa })=81 \quad \mathrm{~V}_{\text {Acion }}(\text { Beta })=50 \quad \mathrm{~V}_{\text {Acion }}(\mathrm{SQ})=25
\end{gathered}
$$

Figura 12. Representação algébrica do processo de cálculo da mensuração da performance das alternativas.

Pode-se, agora, utilizar o modelo para compreender as consequências de cada alternativa neste PVF. Assim, o desempenho das alternativas em análise no PVF - Acionamento foi (Figura 12):

Similarmente foi realizado com os demais PVFs e integrado via Equação global.

$$
\begin{aligned}
& V_{\text {global }}(a)=0,15 V_{\text {Estab }}(a)+0,25 V(a)+0,15 V_{\text {Action }}(a)+ \\
& +0,1 V_{E s p}(a)+0,05 V_{\text {Opcion }}(a)+0,1 V_{\text {Estet }}(a)+0,1 V(a)
\end{aligned}
$$

\subsection{Análise de sensibilidade}

A comparação de alternativas com base em suas performances, tanto locais quanto globais, foi realizada com o auxílio do software HIVIEW. Com este software é possível verificar a resposta do modelo quando da alteração de seus parâmetros, em especial nas taxas de substituição ou no desempenho das ações potenciais (ENSSLIN; MONTIBELLER; NORONHA, 2001).
A Análise de Sensibilidade pode ser tanto gráfica quanto numérica. Um exemplo de Análise de Sensibilidade Numérica encontra-se nos quadros da Figura 13 a seguir, que ilustram esse processo para o PVE - Conforto Térmico.

Como pode ser observada a melhora da alternativa SQ em um nível no PVE- Conforto Térmico provoca um acréscimo em seu desempenho de 12 pontos $(37-25=12)$.

A Análise de Sensibilidade Gráfica, agora considerando o PVE - Controle de Portas. A Figura 14 ilustra que, para o intervalo de $0 \%$ a $34 \%$, a alternativa Alfa permanece como a com maior pontuação. A partir de 34\% a alternativa Beta supera a Alfa.

A Análise de Sensibilidade expande 0 entendimento do decisor sobre a estabilidade ou não da superioridade de uma alternativa em relação à outra, assim, como fornece orientação para o decisor para os fatores em que se tem os aperfeiçoamentos que trariam maior contribuição segundo a percepção dos usuários. 


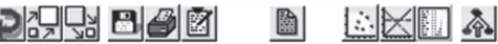

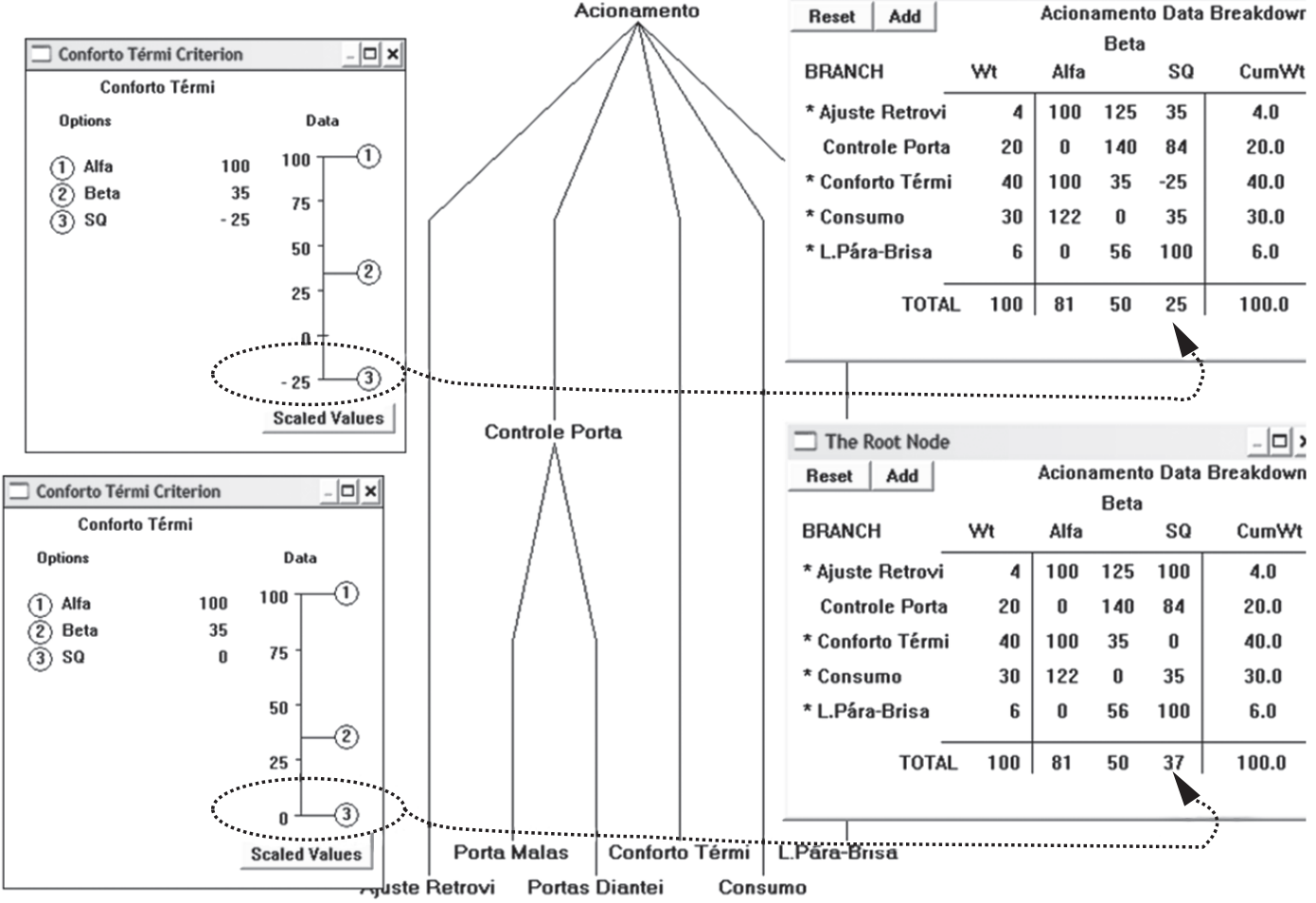

Figura 13. Análise de sensibilidade numérica do PVE- Conforto Térmico para a alternativa SQ quando esta muda seu impacto de N1 para N2.

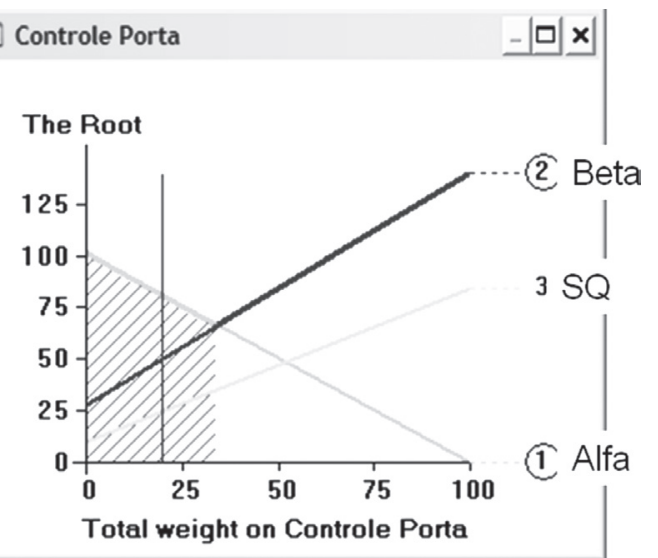

Figura 14. Análise de sensibilidade gráfica do PVE - controle de portas.

\section{Considerações finais}

A abertura dos mercados trouxe como resultado o acirramento da concorrência e via de consequência a necessidade do desenvolvimento de novas formas de ser competitivo. Dentre as dimensões identificadas por Hayes e Pissano (1994), utilizadas pelas empresas de classe mundial para criar diferenciais competitivos: custo, qualidade, flexibilidade, agilidade e inovação, a última tem se destacado como uma das mais promissoras. A dificuldade do uso da inovação sempre teve como limitante o fato de requerer pesadas estruturas de talentos humanos e laboratoriais. Este artigo apresenta uma contribuição a esta dimensão ao mostrar como a metodologia MCDA-C pode ser utilizada como um instrumento para deliberadamente incorporar um processo gerador de oportunidades de inovação em produtos.

0 trabalho evidenciou a forma estruturada utilizada pela metodologia MCDA-C para identificar, organizar, mensurar e integrar aquilo que o usuário (cliente) percebe como importante no produto, aqui denominado como as necessidades do cliente. lsto permitiu a geração de um modelo construído segundo os valores e preferências do usuário e, portanto, por ele legitimado que expandiu seu conhecimento para permitir-lhe entender o impacto do status quo e de alternativas por ele julgadas como possíveis aperfeiçoamentos. 0 modelo desta forma permitiu ao cliente visualizar como suas necessidades foram representadas em um modelo aqui denominado modelo de requisitos do cliente 
e assim compreender como e quanto às possíveis alternativas atenderiam a suas necessidades. 0 modelo de requisitos por sua vez também permitiu ao designer associar as características de qualidade do produto às necessidades do cliente e assim ter um processo para gerar aperfeiçoamentos e visualizar seu impacto no cliente. Como cada um dos requisitos é representado por uma escala que mensura ordinalmente e cardinalmente a performance do produto, esta escala pode ser utilizada para identificar oportunidades para o aperfeiçoamento de propriedades existentes, assim como para identificar as propriedades ainda não incorporadas ao produto e que são desejadas pelo usuário e, assim, inovando. A inovação passa a ser um processo deliberado.

Para o estudo de caso realizado, a aplicação da metodologia MCDA-C deu-se na macro fase de desenvolvimento mais especificadamente no Projeto Informacional, de acordo com a Figura 1, na qual uma das atividades é identificar as necessidades do usuário, convertê-las em requisitos de projeto e, por fim, gerar uma lista de especificações meta para o produto, que é o documento que mostra a alternativa fictícia ideal para o usuário (ROZENFELD et al., 2006) 0 resultado da construção do conhecimento por meio da metodologia MCDA-C explicitou os cenários e os critérios das variações.

0 uso da ferramenta MCDA-C como facilitadora na identificação e hierarquização das características latentes e requisitos dos usuários apresentados e exemplificados em alternativas torna o processo de inovação passível de ser controlado.

A análise com um grupo de três usuários (decisor), ou personagem símbolo em termos de processo de desenvolvimento de produtos como apresentado nesse artigo, tem seus resultados referentes aos direcionamentos do sujeito entrevistado, necessitando assim, para uma aplicação prática, de dados com uma amostragem relevante de parcela considerável do mercado.

A legitimidade do modelo de requisitos desenvolvido foi em todas as etapas de sua construção testada quanto a seu propósito de ajudar as três clientes a melhorar o entendimento de suas necessidades e de como as possíveis alternativas atendiam-nas. Já a validade do modelo de requisitos como instrumento para representar as características de qualidade do produto não foi testada e deixa-se como recomendação para futuros trabalhos esta etapa.

Diante do exposto recomenda-se que, em trabalhos voltados para obtenção de requisitos de projeto, o método MCDA-C seja aplicado em um cenário com vários grupos de decisores. Como por exemplo, utilizado em grupos-foco (focus group), em que o perfil dos participantes é representativo do target pré-determinado. Aumentando assim, a chance do produto em desenvolvimento ser mercadologicamente aceito e, por consequência, obter sucesso.

\section{Referências}

AZEVEDO, J. Aplicação da metodologia multicritério de apoio à decisão na seleção de centros de usinagem para uma central de usinagem. Dissertação (Mestrado em Engenharia de Produção)-Universidade Federal de Santa Catarina, Florianópolis, 2001.

BANA E COSTA, C. A. et al. Decision support systems in action: integrated application in a multicriteria decision aid process. European Journal of Operational Research, v. 113, p. 315-335, 1999. http://dx.doi.org/10.1016/ S0377-2217(98)00219-7

BANA E COSTA, C. A.; DE CORTE, J. M.; VANSNICK, J. C. On the mathematical foundations of macbeth. In: GRECO, J. F.; EHRGOTT, S. M. Multicriteria Decision Analysis: state of the art survey. Boston, Dordrecht, London: Springer Verlag, 2005. p. 409-442.

BARZILAI, J. On the foundations of measurement. In: INTERNATIONAL CONFERENCE ON SYSTEMS, MAN AND CYBERNETICS - IEEE, 2001, Tucson. Proceedings...2001. p. 7-10.

BOMFIM, G. A. Metodologia para o desenvolvimento de projetos. João Pessoa: Ed. Universitária/UFPB, 1995.

BURDEK, B. E. História, teoria e prática do design de produtos. São Paulo: Edgard Blucher, 2006.

EDEN, C. Cognitive mapping: a review. European Journal of Operational Research, v. 36, p. 1-13, 1988. http://dx.doi. org/10.1016/0377-2217(88)90002-1

ENSSLIN, L. et al. Avaliação do desempenho de empresas terceirizadas com o uso da metodologia multicritério de apoio à decisão-construtivista. Revista Pesquisa Operacional, v. 30, n. 1, p. 125-152, 2010.

ENSSLIN, L.; DUTRA, A.; ENSSLIN, S. MCDA: a constructivist approach to the management of human a resources at governmental agency. International Transactions in Operational Research, v. 7, p. 70-100, 2000. http://dx.doi.org/10.1111/j.1475-3995.2000.tb00186.x

ENSSLIN, L.; ENSSLIN, S.; PETRI, S. A importância da estruturação de contextos. Laboratório de metodologias multicritério e apoio à decisão, 2007. p. 1-15.

ENSSLIN, L.; MONTIBELER NETO, G.; NORONHA, S. M. Apoio à decisão - metodologia para estruturação de problemas e avaliação multicritério de alternativas. Florianópolis: Insular, 2001.

ENSSLIN, S. A incorporação da perspectiva sistêmicosinergética na metodologia MCDA- Construtivista: uma ilustração de implementação. 2002. 478 f. Tese (Doutorado em Engenharia de Produção)- UFSC, Florianópolis, 2002.

GOMES, C. F. S.; GOMES, L. F. A. M. A função de decisão multicritério. Parte 1: Dos conceitos básicos à modelagem multicritério. Revista do Mestrado em Administração e Desenvolvimento Empresarial, v. 2, n. 3, 2007. 
HAYES, R. H.; PISANO, G. P. Beyond world-class: the new manufacturing strategy. Harvard Business Review, v. 72, n. 1, p. 77-86, 1994.

KEENEY, R. L. Value-focused thinking: a path to creative decisionmaking. Cambridge, MA: Harvard University Press, 1992.

KINDLEIN JÚNIOR, W. Analogia entre as metodologias de desenvolvimento de produtos atuais, incluindo a proposta de uma metodologia com ênfase no ecodesign In: CONGRESSO INTERNACIONAL DE PESQUISA EM DESIGN, 2., 2002, Brasília, DF. Anais... 2002.

LANDRY, M. A note on the concept of problem. Organization Studies, v. 16, p. 315-343, 1995. http://dx.doi. org/10.1177/017084069501600206

LARSON, E. W.; GOBELl, D. Organizing for product development projects. Journal of Product Innovation Management, 1988. OXFORD. 05.

LIMA, M. V. A. Metodologia construtivista para avaliar empresas de pequeno porte no Brasil, sob a ótica do investidor. 2003. 382 f. Tese (Doutorado em Engenharia de Produção)- UFSC, Florianópolis, 2003.

PARATH, R. et al. Um modelo multicritério em apoio à decisão integrado a um sistema de gerência de passivos ambientais de uma rede rodoviária. In: ENCONTRO NACIONAL DE CONSERVAÇÃO RODOVIÁRIA, 10., 2005, Joinvile, SC. Anais... Joinvile: ENACOR, 2005.

ROBERTS, F. S. Measurement theory. Addison-Wesley, 1979.

ROY, B. Multicriteria methodology for decision aiding. Dordrecht: Kluwer Academic Publishers, 2006. http://dx.doi.org/10.1016/0377-2217(93)90312-B

ROY, B. Decision science or decision-aid science? European Journal of Operacional Research, v. 8, n. 1, p. 184-203,1993.

ROY, B.; VANDERPOOTEN, D. The european school of MCDA: emergence, basic features and current works. Journal of Multi-Criteria Decision Analysis, v. 5, p. 22-38,1996.

ROZENFELD, H. et al. Gestão de desenvolvimento de produtos. São Paulo: Saraiva, 2006.

SKINNER, W. The productivity paradox. Management Review, v. 75, p. 41-45, 1986.

ZIMMERMANN, H. An application-oriented view of modeling uncertainty. European Journal of Operations Research, v. 122, p. 190-198, 2000. http://dx.doi.org/10.1016/ S0377-2217(99)00228-3

\title{
ldentification of costumers needs in the Products Development Process: an innovative proposal illustrated for the automotive industry
}

\begin{abstract}
Among the winning strategies in today's competitive environment innovation, one emerges as of the most successful - the ability to identify and measure customers' needs, which is perhaps the greatest challenge for designers. In this context, this paper suggests a proposal aiming to help identify customers' needs and transform them into requirements and quality characteristics to support decision making in the phase of product development. In order to test this proposal, a scenario has been created and the degree of meeting the needs of a particular user when purchasing a vehicle for private use has been evaluated. This paper presents a case study of primary non-obstructive sources of information, qualitative and quantitative variables, and a constructivist view of knowledge. The proposed procedure allowed the designer to identify and measure the quality characteristics and requirements of the needs perceived by the customers.
\end{abstract}

Keywords

Design. Customer requisites. MCDA-C. Performance measurement. Decision. 